BULLETIN Bulletin hispanique

HISPANIQUE Université Michel de Montaigne Bordeaux

112-1| 2010

Actes du Colloque « langue, littérature, littéralité »

\title{
Histoire d'amour
}

Le Sujet d'écriture et la Littéralité

\section{Michèle Ramond}

\section{(2) OpenEdition \\ Journals}

Édition électronique

URL : http://journals.openedition.org/bulletinhispanique/1166

DOI : 10.4000/bulletinhispanique. 1166

ISSN : 1775-3821

Éditeur

Presses universitaires de Bordeaux

Édition imprimée

Date de publication : 1 juin 2010

Pagination : 359-371

ISBN : 978-2-86781-692-5

ISSN : 0007-4640

Référence électronique

Michèle Ramond, "Histoire d'amour », Bulletin hispanique [En ligne], 112-1 | 2010, mis en ligne le 01 juin 2013, consulté le 20 avril 2019. URL : http://journals.openedition.org/bulletinhispanique/1166 ; DOI : 10.4000/bulletinhispanique. 1166 


\title{
Histoire d'amour Le Sujet d'écriture et la Littéralité
}

\author{
Michèle Ramond \\ Université Paris 8
}

À la frontière de la littéralité et de l'inconscient du texte, le pan de mur atteint par le soleil dans la cour de la maison d'Eugénie Grandet et la forge visitée par la lune du célèbre "romance " de García Lorca vont nous servir de métaphores pour exprimer l'expérience subjective réfléchie par les textes que nous avons le plus aimés et qui continuent jour après jour de nous absorber. Cette illumination de nos cuvres favorites par une intériorité secrète, qui n'appartient qu'au texte qui la transmet, transforme la littérature en un événement esthétique toujours unique. C'est cet émerveillement que nous tentons ici de dire, en hommage à Nadine Ly.

En la frontera entre la literalidad y el inconsciente del texto, el trozo de muro iluminado por el sol en el patio de la casa de Eugenia Grandet y la fragua visitada por la luna en el famoso romance de García Lorca nos sirven de metáforas para expresar la experiencia subjetiva reflejada en los textos que más hemos querido y que nos siguen absorbiendo día tras día. Esta iluminación de nuestras obras predilectas por una interioridad secreta, que solamente pertenece al texto que la transmite, transforma la literatura en un acontecimiento estético siempre único. Este deslumbramiento es lo que intentamos aqui transmitir a modo de homenaje a Nadine Ly.

On the border between literality and unconscious in the text, the section of wall reached by the sunlight in Eugénie Grandet's courtyard and the moonlit smithy in Garcia Lorca's famous ballad, will be used as metaphors in order to express the subjective experience induced by the texts we love most, which, day after day, unendingly entrance our minds. The illuminating power of our favourite books by a secret interiority, which exclusively

$B H i$, Tome 112, n $^{\circ} 1$ - juin 2010 - p. 359 à 371. 
belongs to the textual-artefact, converts literature into an altogether inimitable aesthetic event. This rapture of ours, which we try to analyse in this essay, is meant as an homage to Nadine Ly.

Mots-clés : Sujet d'écriture - Littéralité - Événement esthétique - Inconscient du texte.

Quand le soleil atteignit un pan de mur, d'où tombaient des Cheveux de Vénus aux feuilles épaisses à couleurs changeantes comme la gorge des pigeons, de célestes rayons d'espérance illuminèrent l'avenir pour Eugénie, qui désormais se plut à regarder ce pan de mur, ses fleurs pâles, ses clochettes bleues et ses herbes fanées, auxquelles se mêla un souvenir gracieux comme ceux de l'enfance. Le bruit que chaque feuille produisait dans cette cour sonore, en se détachant de son rameau, donnait une réponse aux secrètes interrogations de la jeune fille, qui serait restée là, pendant toute la journée, sans s'apercevoir de la fuite des heures. Puis vinrent de tumultueux mouvements d'âme. Elle se leva brusquement, se mit devant son miroir, et s'y regarda comme un auteur de bonne foi contemple son œuvre pour se critiquer, et se dire des injures à lui-même.

Je ne suis pas assez belle pour lui.

(Honoré de Balzac, Eugénie Grandet, 1833)

T OBJEт esthétique atteint-il quelque jour la perfection et, à supposer 1 même que cela soit possible, à quoi tient cette perfection ? À la qualité même de l'objet esthétique dont il faudra nous expliquer à quoi elle se mesure, ou à la nature de la communication esthétique dont cet objet est le médium, c'est-à-dire à l'événement esthétique ?

Pour ne parler que de la littérature, il y a bien une "dynamique opérative » (j'emprunte ce concept à Nadine Ly) qui traverse la matière textuelle et qui nous la rend plus ou moins proche et enthousiasmante. Toutes les matières textuelles, dans le cas contraire, se ressembleraient, elles sont toutes faites de mots et d'énoncés. Si nous sommes plus ou moins transportés ce n'est même pas en fonction de la richesse du vocabulaire ou de la rareté de son ordonnancement syntaxique. Être surpris n'est pas être séduit, être séduit, c'est être saisi, au cours de la lecture, par un événement esthétique qui se trouve en quelque sorte enchâssé dans l'objet esthétique, pour nous dans le texte. 
Un peu à la façon d'une énergie invisible qui traverse la matière textuelle, bouleverse ses énoncés, transfigure leur sens au-delà de l'imaginable.

Cette sorte d'aliénation, de transport, d'extase que produit en nous telle transgression métaphorique, ou tel déplacement métonymique, ou telle figure hybride ou équivoque accompagne la prouesse stylistique mais suppose aussi autre chose car la prouesse seule n'éveillerait que l'agrément d'une surprise vite remplacé par la lassitude qu'engendre, à la longue, tout artifice. Or l'objet esthétique qu'est pour nous le texte atteint la perfection quand il engendre le rêve du lecteur, mieux encore quand il devient son rêve :

je ne suis pas assez belle pour lui.

Mon temps est suspendu et mon espace perd sa ponctualité, ma quotidienneté se dissout dans l'urgence du texte qui me requiert toute, je suis dans son éblouissement et sous son emprise. Enveloppée dans son énergie noire, je vais me concentrer sur sa dramaturgie, car tout me laisse supposer que le charme dont je suis frappée, qui m’ébranle, a son origine dans les couches profondes du texte. La littéralité puiserait-elle sa lumière dans une autre île que celle du texte, dans une Atlantide, un lieu sans lieu, un lieu souterrain ou ruiné ou perdu dont la mélancolique beauté relève la médiocrité de nos occupations et de nos préoccupations, de nos travaux et de nos jours?

Longtemps j'ai pensé que la chose extraordinaire des textes prestigieux qui enchantent nos vies, c'était la présence en eux, dans leurs limbes ou leurs cryptes, d'une dramaturgie inconsciente dont la glorieuse remontée à la surface de la littéralité constituait l'événement esthétique. La matière textuelle se trouve alors transfigurée par ses profondeurs, hypothèse d'un soubassement du sens, d'un sens inaccessible, pratiquement impossible à restituer dans son intégralité, qui provoque l'attente indéfinie du lecteur et même son inattention au sens lexical et grammatical. L'objet esthétique, le texte, s'impose dans cette hypothèse comme un être prégnant, l'événement esthétique illumine et disperse le statut de l'objet. Mais l'événement esthétique est aussi insaisissable et plein d'émotions incontrôlables que le pan de mur atteint par le soleil que contemple Eugénie et qui illumine son avenir de célestes rayons d'espérance. Pour aussi attentifs que nous soyons à ce mur et aux plantes qui le couvrent sous le beau soleil des automnes naturels aux rives de la Loire, nous ne comprendrons pas les souvenirs gracieux qui s'y mêlent ni les secrètes interrogations, ni les tumultueux mouvements d'âme qui le parcourent. Notre appréciation de l'objet esthétique nous conduit à 
ne pas l'écarter de son contexte culturel, historique, érotique, mais nous ne pouvons repousser une autre tentation, celle de nous laisser complètement absorber par lui, d'oublier même son caractère esthétique ou plutôt de ne voir dans cette propriété qui ne saurait lui être refusée que l'offre d'un passage vers une expérience subjective réfléchie par le texte. Sans qu'un jugement de valeur sur le texte ait à intervenir, nous serons ou non frappés par l'éclat de la lumière qui le transfigure, dont l'éblouissement nous atteint et qui transforme notre vision. Et nous apercevrons alors, enchâssés dans son discours, au bruit rythmé de ses vers ou de ses phrases, tant de souvenirs d'enfance, de secrètes interrogations et de tumultueux mouvements d'âme que l'expérience vécue de la lecture, en se prenant à réfléchir sur son extase, essaiera d'en élaborer une représentation. Dans un commentaire raisonné ou nostalgique, elle évoquera l'événement esthétiqueà la façon d'une illumination par l'intériorité secrète du texte qui tout aussi bien aurait pu se taire ou se régler sur d'autres exigences et d'autres discours. Il se trouve pourtant que telle intériorité secrète s'est emparée lexicalement et grammaticalement de tel texte et point d'un autre et qu'au cœur même de ce texte particulier elle opère comme un acteur incliné à relater des souvenirs d'enfance, à confier des interrogations secrètes et de tumultueux mouvements d'âme qui sans le texte n'auraient de fait pas existé :

je ne sais pas si je suis assez belle pour lui.

Pour en revenir au fameux pan de mur d'où tombaient des Cheveux de Vénus, touché par la grâce d'un gai soleil matinal d'automne mais illuminé aussi par la rêverie d'une jeune fille qui le peuple de ses imaginations, celui-ci s'empare, sans que je puisse en arrêter la magie, de ma propre imagination et je ne peux m'empêcher de rêver en sa compagnie à l'événement extraordinaire qui traverse les textes que nous aimons. Nous avons tous nos pans de mur et nous y retournons comme à des figures insolites et tentatrices dont nous suivons, jusqu'àl'aveuglement, les pleins et les déliés. Surl'axe del'horizontalité nous attendons avec une anxiété enfantine troublée de mauvais présages le mariage d'Eugénie avec Charles Grandet. Sur l'axe de la verticalité nos espérances en revanche grandissent avec le temps et les nouvelles relectures nous font effectuer mille opérations d'alpinistes; une chute nous arrête parfois à mi-chemin mais nous effectuons aussi des remontées glorieuses, cependant aucun commentaire vaguement philosophique ne pourra épuiser l'objet esthétique illuminé de soleil, d'espérances et de rêves dont la vision ne nous décevra jamais mais ne nous comblera pas non plus car le véritable objet de notre quête nous sera refusé. Que cherchons-nous exactement dans le pan 
de mur miroitant où le soleil du matin d'automne se mêle aux Cheveux de Vénus à couleurs changeantes, aux souvenirs d'enfance et aux rêveries d'Eugénie et aux nôtres tout aussi fous, irraisonnés ou déraisonnables ? Que cherchons-nous (qu'avons-nous autrefois cherché) dans la forge obnubilée par l'astre lunaire qui la transforme en miroir, en pan de mur glacé par la nuit où l'aspect des choses ordinaires se transforme, devient vague et inexplicable ? Quel trouble y avons-nous rencontré que nous ne savons pas, aujourd'hui encore, justifier?

Ce pan de mur, cette forge, qui provoquent notre attente, nous enveloppent dans leur lumière : un jour pur et le beau soleil des automnes naturels aux rives de la Loire, une nuit pure et la pleine lune naturelles aux chromos de la montagneuse région grenadine. Le pan de mur et la forge nous enveloppent dans leur silence insolite, les arabesques décrites par la lune qui danse sur la paroi rupestre de la forge et les Cheveux de Vénus aux feuilles épaisses et aux couleurs changeantes qui retombent sur le pan de mur nous font des signes indéchiffrables. Les couleurs changeantes comme la gorge des pigeons, les seins blancs en dur étain, les fleurs pâles, les clochettes bleues, les herbes fanées, les bras qui battent l'air, le jupon amidonné qui fleure le nard décrivent, sur le pan de mur et sur la paroi rupestre, de gracieuses volutes faussement prometteuses d'avenir radieux, affolantes et répressives où le regard fasciné se perd en rêveries, en secrètes interrogations, retourne aux souvenirs de l'enfance. Puis viennent les tumultueux mouvements d'âme de la jeune fille et de l'enfant qui se mirent dans le miroir textuellement impassible, plein de secrets et d'énigmes, du pan de mur ensoleillé ou de la paroi lunaire. On resterait là, nous aussi, pendant toute la journée ou toute la nuit, sans s'apercevoir de la fuite des heures ou des années. Le temps passe et le mystère demeure, inscrit parmi les Cheveux de Vénus et les arabesques de la Gitane sur le mur de la cour et la paroi de la grotte où l'astre du jour et celui de la nuit nous égarent. Confondus dans la modestie et la crainte de la jeune fille et de l'enfant, à notre tour nous nous levons et nous nous mettons devant le miroir du mur de la cour ou de la forge et nous nous y regardons comme un auteur de bonne foi contemple son œuvre, le texte spéculaire et incertain qu'un énigmatique destin purifie :

je ne suis pas assez belle pour lui.

Le pan de mur subitement éclairé par un beau soleil d'automne avait-il prévu le lent malheur de la solide mais belle jeune fille, devenue à trente trois ans un des meilleurs partis de Saumur, belle encore " mais comme une femme est belle à près de quarante ans ", riche veuve mais vouée à une passion 
sans espoir inspirée par un homme qui certes ne la méritait pas ? Habituée au malheur depuis son enfance dans la maison de Saumur sans soleil, sans chaleur et sans cesse ombragée, de quels désirs et de quels rêves la fille du tonnelier avait-elle peuplé ce pan de mur où son regard toute la journée s'est abîmé ? Généreuse et charitable, Madame de Bonfons inspire généralement un religieux respect et il n'y a que le mur d'où tombaient des Cheveux de Vénus pour se rappeler encore les secrètes interrogations amoureuses et les tumultueux mouvements d'âme d'Eugénie dans la splendeur de sa jeune beauté de Vénus de Milo certes un peu masculine mais ennoblie par une chaste vie et la suavité du sentiment chrétien. L'argent qui faisait tout le bonheur du bonhomme Grandet communique peu à peu ses teintes froides à Eugénie et semble la priver des sentiments pour lesquels elle était pourtant faite, étant elle-même toute sentiment. Le pan de mur seul en garde la mémoire et nous la restitue par ce texte magnifique qui en est la version littérale, Eugénie Grandet. Mais à qui appartient la tristesse ou la mélancolie de ce froid qui gagne la vie, de cette ombre glaciale, de ces teintes froides qui enlèvent à la vie céleste d'Eugénie son éclat comme autrefois une petite vérole avait gâté chez la jeune fille le velouté de la peau néanmoins douce et fine encore ? Le texte comme le pan de mur a besoin de la pâle et triste enfance d'Eugénie pour lui faire miroiter un amant auquel elle donne son cœur parmi le bruit léger des feuilles d'automne qui tombent dans la cour, mais qui ne lui causera que des douleurs mêlées de frêles et bien inutiles espérances.

Le pan de mur automnal et le texte qui en est le grossissement littéral recueillent le lent refroidissement des illusions enfantines et des émois érotiques de la jeune fille. L'automne ensoleillé qui ruisselle sur le mur de la cour où l'âme d'Eugénie s'infiltre et s'enchâsse se mue en hiver glacé où le seul sentiment qui subsiste est l'amour qui unit Eugénie à sa vieille Nanon. Mais qui est Eugénie ? Qui est Charles ? Qui se languit en se regardant dans le miroir, qui se critique en contemplant son œuvre ou son reflet, et de quel dépérissement d'âme cette inquiétude critique est-elle l'image, de quel esprit mélancolique cette image qui déçoit son auteur est-elle la lettre ?

$\mathrm{Si}$ je fais ici intervenir la notion de «sujet d'écriture » j'en éprouve quelque effroi comme celui ou celle qui retrouve brusquement dans un tic de sa main, ou dans quelque autre manie qui n'avait pas auparavant attiré son attention, la copie exacte et bien sûr involontaire d'un geste habituel de sa mère ou de son père disparus. La notion de " sujet d'écriture " appartient à un âge de la vie où elle m’apparut, dans sa splendeur freudienne, comme l'heureux augure d'une instance nouvelle, à la fois littéraire et psychique. Un narrateur n'ayant pas à témoigner de sentiments et l'auteur se tenant 
suffisamment en retrait de son ouvre pour ne pas tomber dans son texte, faire corps avec lui, et d'une certaine façon compromettre dans cette chute sa survie littéraire, je pensai alors que la neutralité sentimentale du narrateur et le silence insolite et pudique mais forcément nécessaire de l'auteur ouvraient passage à la remontée glorieuse d'un autre sujet qui avait textuellement tous les droits.

Cet autre sujet ou ce sujet psychotextuel installé dans le temps et l'espace réglés du texte m'apparaissait comme l'âme du texte ; inséparable du corps du texte, il devenait le véritable objet esthétique de ma contemplation, celui que j'assimilais au texte, à sa littéralité confuse et bruissante, mais auquel, cédant à son désir, je reconnaissais aussi une capacité humaine. Le texte dont la lecture et l'analyse me rendaient accessible cette fonction subjective, inséparable de sa littéralité, me livrait en quelque sorte l'intimité de sa fabrique. L'objet esthétique, le texte littéraire, était devenu la forme figurative des désirs et des angoisses du sujet d'écriture. Sorte de tautologie qui consistait à voir dans le texte son sujet ou d'aimer le sujet à travers la forme figurative de son texte. Forme figurative et sujet d'écriture se renvoyant l'un(e) à l'autre dans un dialogue perpétuel qui agitait le corps textuel sous l'effet des émotions concomitantes éprouvées par son sujet. Il y avait donc pour moi syncrétisme de l'objet esthétique et du sujet d'écriture, les deux actants se trouvant fusionnés au point même qu'une lecture superficielle du texte donnait consistance à cette instance subjective solidaire du texte, rapprochée de ses moindres détails mais délestée des fardeaux de l'auteur, de ses scrupules et de ses contraintes. Il pouvait se faire cependant que l'émotion plus vive ou plus émotive de l'auteur se trouvât elle aussi impliquée dans la littéralité effusive du texte, distribuée à la fois sur la forme figurative et sur la fonction subjective qui animait cette forme et qui justifiait tout le texte. Il s'agissait alors de faire la différence entre ces divers niveaux de sentiment et d'émotion. Le point de vue de l'observateur devenait plus que jamais une instance du texte, une pièce essentielle de l'objet esthétique, servant à discriminer non pas le vrai du faux, le texte contenant en lui-même toutes ces valeurs, mais le rêve immobile de l'auteur errant à la surface du texte, du mouvement subjectif inattendu qui avait eu la grâce d'accompagner à toutes ses étapes la forme figurative du texte qui nous charmait.

Grâce à ce geste d'évaluation de l'observateur, des ordres subjectifs différents et successifs affectaient le texte, l'un qui cédait à une approche de type cognitif et qui concernait plutôt l'émotivité de l'auteur, l'autre en conjonction tellement totale avec la littéralité du texte qu'il était impossible de l'en extraire comme on extrait le sens d'un mot. Au contraire, ce sujet intérieur et la forme figurative de son angoisse et de son désir ne faisaient 
qu'un, on ne pouvait analyser la forme sans aborder aux territoires du sujet d'écriture, fusionné avec elle, tandis que les émotions et les sensations émanant de l'auteur n'agitaient le texte que superficiellement, de façon parfois pathétique tellement elles étaient sincères et volubiles, mais sans faire corps avec le texte. C'est ainsi que la passion d'un sujet en quelque sorte extérieur au texte et flottant au-dessus du texte, sans entrer en conjonction secrète avec sa littéralité, pouvait se discerner lors d'une lecture superficielle. Mais ce sujet flottant, à la dérive, porté par le texte sans pour autant concerner sa fabrique et sa facture, n'était pas ce qui m'apparaissait comme le sujet d'écriture, ce mouvement subjectif qui animait la littéralité et qui constituait son intériorité sensorielle et psychique sans l'aide d'aucune médiation extérieure et référentielle.

Abîmée dans la contemplation du pan de mur que le soleil automnal vient d'atteindre, de ses Cheveux de Vénus, de ses feuilles, de ses fleurs, de ses clochettes, de ses herbes fanées, Mademoiselle Grandet est en communication avec la subjectivité intérieure du texte, avec les tumultueux mouvements d'âme où la littéralité s'incline, avec le rêve de mots que l'illusion amoureuse d'Eugénie ne décrit pas dans toute sa magnitude. Pour arriver, si tant est qu'on le puisse, au secret du drame qui se joue dans le pan de mur frappé par le soleil d'automne et dans tout le roman dont le pan de mur offre la fulgurante synthèse, il faudrait réaliser une saisie esthétique totale et exceptionnelle de tous les événements et de toutes leurs articulations, morceau par morceau de toute la matière narrative du texte.

Cette saisie sans doute impossible de toute la littéralité d'Eugénie Grandet nous permettrait de parvenir au bruit rythmé d'une subjectivité aussi essentielle à la forme textuelle qu'interdite à l'observateur. Au cœur du sujet d'écriture de ce grand roman, on verrait s'abattre sur une jeune fille point assez belle, sur son désir craintif et sur sa modestie, l'ombre glaciale du père tonnelier et de sa maison.

Eugénie Grandet n'est pas le sujet d'écriture, ni Madame de Bonfons, ni la future (probable) Madame de Froidfond, et pourtant cette jeune fille bien née que son doux tempérament et son nom semblent destiner au bonheur d'épouse et de mère sera entravée et amoindrie par les petitesses et coutumes de sa vie première, la froide toile de fond de la maison de Saumur et le mauvais fond du tonnelier, préoccupé seulement de fonds, de capital et de revenu. Il se mélange sur la palette de l'héroöne Grandet les bienfaits du ciel et les intérêts et bassesses du monde, faire son salut au milieu des trahisons et des égoïstes commerces réclame le secours des plus beaux ornements. De cette lutte à mort, l'œuvre est le fruit. 
On comprend mieux à présent, peut-être, le sens de cette approche prudente de l'objet esthétique. Sa surface phénoménale, inépuisable, cache un drame dont nous mesurons le progrès et les variations, le sujet d'écriture peut se comparer aux mouvements eustatiques de la mer, il est virtuellement contenu dans tous les événements, toutes les palpitations et toutes les couleurs du texte, son point d'intimité cependant se rapproche d'une figure génératrice, née pour lui servir de point d'appui, de fond inspirateur. L'histoire du texte est l'histoire de cette rencontre bienheureuse entre la figure inspiratrice et le sujet d'écriture virtuel, en cours d'avènement textuel. Ici Eugénie est, de toute évidence, la première représentation du sujet d'écriture, elle est la figure par qui le sujet textuel devient saisissable, elle rayonne l'énergie subjective du texte, elle est sa matérialité émouvante. Mais cette merveilleuse conjonction, dont le sens est inépuisable, a tôt fait de s'évanouir si l'on envisage Eugénie comme un personnage de fiction, elle n'exhale l'énergie du texte que si nous considérons Eugénie et le roman auquel elle donne son nom comme une totalité constituée, indivisible. Le sujet d'écriture est dans l'inséparabilité d'Eugénie, de sa généalogie, de la maison Grandet et de la maison textuelle, il a pour corollaire une attitude analytique attentive à la totalité esthétique constituée.

Le dialogue secret d'Eugénie avec le pan de mur ensoleillé d'où tombaient des Cheveux de Vénus aurait pu durer toute la journée si la jeune fille ne s'était levée brusquement pour se mettre devant son miroir et constater qu'elle n'était pas assez belle pour lui. Mais en réalité ce dialogue secret se transporte à tout le texte du roman devenu à la fois le pan de mur atteint par le soleil d'automne des bords de Loire et le miroir où, comme l'auteur de bonne foi, Eugénie contemple son œuvre. L'hybridité d'Eugénie ne saurait mieux se dire. Inséparable de la maison de Saumur, de son jardin et de sa cour, elle est également inséparable du texte où elle se mire et s'évalue, nous renvoyant l'image du laid que cache mal la beauté, du laid qui gâche la beauté, mélange explosif de bleu céleste et de noir, ce noir qui gâche toute la beauté du monde. Ce gâchis déjà se devine sur le visage rond d'Eugénie, " jadis frais et rose ", dont une petite vérole a abîmé les traits, dans sa tête énorme qui nuit un peu à sa ressemblance première avec la Vénus de Milo, et sur le pan de mur que le soleil d'automne finit par atteindre, mais que les herbes fanées et le bruit des feuilles mortes tombant bruyamment dans la cour colorent d'un fond de désenchantement où la décomposition des rêves de la jeune fille se prédit. C'est bien dans l'interférence indémêlable de toute l'énergie et du bonheur du monde et de la conviction que tout aboutit à la décomposition, que se situe le sujet d'écriture ; c'est sur ce fond fertile en rêves gracieux et tumultueux mais surtout en souffrances que le sujet 
rayonne dans la moindre parcelle esthétique de son texte. Il s'agit, on le voit, non de nuire à la splendeur de la littéralité du texte, mais bien de se laisser enchanter par elle en laissant l'expérience esthétique se transformer peu à peu en une expérience amoureuse, comme si on se disposait à assumer tout le désir et la douleur de son sujet :

mais je ne suis pas assez belle pour lui.

Le "Romance de la luna, luna » (1926) de García Lorca est une composition pour violon et percussions qui propose mais aussi qui raconte une expérience esthétique convertible à chaque instant en expérience amoureuse. L'illusion romanesque est aussi forte dans ce poème que tout à l'heure dans le roman d'Eugénie Grandet. Mais la manifestation figurative est réduite à sa plus simple expression, ce qui n'implique pas qu'elle soit moins complexe que dans le roman. Nous avons fait l'économie apparente de soixante-dix pages de texte et nous nous trouvons dans la situation de rêverie et d'hypnose qui fut aussi la nôtre quand Eugénie se mirait, non encore dans son miroir qui fit injure à ses espérances amoureuses, mais dans le pan de mur éclairé de soleil où un souvenir gracieux comme ceux de l'enfance tint son regard captif pendant presque toute la journée. C'est ici la forge qui fait office de pan de mur ou de caverne de Platon, et c'est sur ce miroir rupestre, qui nous parle déjà de fabrique poétique, que l'enfant de la forge contemple son fantôme favori, sa revenante, la pleine lune qui danse, lubrique et pourtant pure comme une mère qui donne le sein, enveloppée dans la lumière lactescente d'un clair de lune gitan. Ce que voit l'enfant resté seul dans la grotte réverbérante de la forge est un objet apparemment plus explicite que le "souvenir gracieux comme ceux de l'enfance ", contemplé par Eugénie sur le fameux mur de la cour plutôt funèbre de la maison de Saumur. Cet objet identifié à une lune qui danse provoque un trouble similaire à celui que ressent la jeune fille devant son énigmatique pan de mur ensoleillé, pleine de ses secrètes interrogations, puis soulevée par de tumultueux mouvements d'âme qui la font se lever brusquement pour se regarder dans son miroir. L'enfant de plus en plus agité, absorbé par la Vénus lunaire qui lui rend visite à la forge, n'a pas à se lever brusquement pour se mettre devant son miroir, la lune du "Romance " est tout à la fois le pan de mur atteint par la lumière, les Cheveux de Vénus, les rayons d'espérance, le souvenir gracieux de l'enfance, le bruit des feuilles mortes qui tombent dans la cour et le miroir d'Eugénie. Les secrètes interrogations et les tumultueux mouvements d'âme soulèvent l'enfant hypnotisé par la visiteuse à la forge et par sa danse autant que par les images secrètes que le miroir lunaire lui 
renvoie. Tout baigne dans une lumière Hoffmannienne et l'enfant est transi d'une peur mythologique où tous les fantasmes érotiques conjuguent leur effroi. Comme la cour, la forge est sonore de tous les bruits érotiques que la lune convoque, un attirail de batteur nous assourdit depuis le crissement du jupon amidonné, l'air frotté par le mouvement des bras de Vénus, les bruits terrifiants de percussions des gitans perçant le disque lunaire qu'ils ont couché sur l'enclume, jusqu'au martèlement de leur chevauchée sur la plaine. Dans cet univers sonore esthétique qui nous communique son épouvante et son tintamarre métallique, l'archer d'alto, mais malhabile, de l'enfant fait vibrer avec de larges frémissements tout le pentacorde (cinq quatrains d'octosyllabes) de cette première étape du "Romance » : " Huye luna, luna, luna ».

Un sujet a-t-il cherché ici à poétiser sa vie d'antan, son intimité avec l'objet maternel imaginaire, l'effroi du sexe ? A-t-il cédé à la tentation de se laisser absorber tout entier par le fantasme? Ou, au contraire, a-t-il ébauché un mouvement de retrait, tente-t-il de s'éloigner, de se retirer, de se dérober à l'illusion de scène amoureuses régressives ?

Curieusement, un nouveau sujet semble faire son apparition dans la deuxième étape du poème, décidé à mettre fin à toutes les tensions pathétiques héritées de l'enfance, à s'évader de la structure polémique de l'intrigue oedipienne sans fin. Encore fallait-il, pour abandonner vraiment cette dimension tragique de l'érotisme enfantin, éliminer le personnage principal du roman familial, le plus attachant, l'amère image de soi que le miroir lunaire ne cesse de renvoyer au sujet, l'impossible enfant au sein, celui qui ne peut plus être : "Niño, déjame».

Encore fallait-il ce meurtre de l'enfant. Celui-ci est permis, dans l'énoncé, par la lente arrivée du jour qui mettra fin aux sortilèges de la lune et de la nuit. Le cavalier solaire s'élève au-dessus de l'horizon, il fait trembler toute la plaine, c'est lui désormais le grand batteur, batteur d'or et de fer et de tambour, le grand percussionniste, percutant il casse le "Romance " en deux moitiés inégales; on perd de la matière textuelle et familiale, on perd un quatrain, l'enfant est éliminé de la forge et la lune aussi, ils cheminent tous deux à présent, la main dans la main, au ciel des illusions perdues. La nouvelle dimension figurative fait de la scène amoureuse d'antan une réalité perdue, le chant de la hulotte, la chouette des bois, établit l'empire de la déesse des Lettres et des Arts, chargée d'un rôle éthique elle accompagne le retour à la forge des gitans-forgerons, quant au sujet esthète qui faisait corps avec l'enfant, ayant franchi le seuil de la forge il accueille finalement la forge en son sein. La saisie esthétique de l'objet est celle du sujet lui-même dans sa dimension tragique, sacrificielle, puisqu'il a renoncé aux délices et 
épouvantes du roman familial, dans sa dimension matricielle également puisqu'à présent c'est lui qui, élargi à tout le paysage évoqué par le texte, contient la forge. Le sujet s'est élargi à la surréalité esthétique du texte, il n'est plus dans la forge, lié au corps et aux émois de l'enfant, mais hors de la forge et il touche les bornes, après ce passage obligé par la mort de l'enfant, du cadre spatial et extatique du "Romance".

L'efficacité suprême du sujet d'écriture, c'est d'être à la fois l'histoire de ce renoncement au souvenir gracieux mais troublant de l'enfance, l'événement esthétique que la forme figurative recouvre, mais aussi tout le signifiant poétique, purifié des transes d'un sujet proche de l'Auteur ou d'un sujet inconscient. Le sujet d'écriture est plein de l'illusion d'une subjectivité inédite, convoquée par le texte à l'instant même où il s'écrit et où il se lit, puis s'abolissant avec lui, suspendue à l'espoir d'une nouvelle assomption esthétique. La vie extatique et fugace du sujet d'écriture, limitée à l'espace clos du poème, tient toute aux tensions pathétiques exercées sur lui par la forge. Le domaine du beau concerne d'abord seulement la forge, mais au fur et à mesure que les gitans s'en approchent, il s'étend alentour à l'horizon d'où surgit le cavalier, à la plaine que son cheval foule, à l'oliveraie que traversent les gitans somnolents, à l'arbre où l'oiseau de Minerve chante, au ciel où, semble-t-il, la lune est retournée tenant un enfant par la main. La forge désormais n'est plus qu'un élément de l'immense paysage avec lequel se confond le sujet, c'est tout cet espace élargi qui est nécessaire au poème comme l'air qu'on respire est nécessaire à la vie. De la forge, nous n'avons plus qu'une saisie esthétique extérieure, des sanglots et des cris nous parviennent, attribués par le poème aux gitans qu'à notre tour nous imaginons plongés dans un deuil irrémédiable, englobés par la quotidienneté de la mort. Mort le petit Éros, la hiérarchie instituée par l'ordre généalogique s'inverse dans un antre poétique où l'enfant-roi impose sa loi aux gitans forgerons, aux maîtres de la forge, de la forgerie imaginaire et de la fabrique poétique. Le lieu métaphorique, inquiétant et mystérieux, n'est pourtant plus sur le devant de la scène, il est passé au second plan et c'est maintenant le rythme respiratoire de l'air qui donne au poème sa profondeur et qui signifie toute son intimité. Installé dans l'air, un sujet Pantocrator à l'écoute de la forge, de sa funèbre musique et de son mystère la tient sous sa protection, sans qu'on puisse rien connaitre du drame qui s'y déroule.

L'attente exaltante mais à jamais retardée d'un épilogue produit l'illusion d'un sujet destinateur inexorable qui crée un monde selon sa logique et ses désirs, mais que nous ne pourrons jamais atteindre. La fatalité et la mort le menacent, comme elles menacent aussi tous les autres personnages intermédiaires, l'enfant, la lune et les gitans, cette trinité familiale iconisée qui 
fera la fortune de l'écrivain García Lorca, mais dont le statut symbolique est tragiquement instable. Le sujet Pantocrator qui organise l'ordre du monde, le mystère de ces neuf quatrains octosyllabiques, hésite entre des choix d'amour différents, tresse des réseaux de complicité entre des objets inconciliables, confond l'union des corps avec la mort, privilégie érotiquement tantôt un acteur tantôt un autre. Tantôt il devient pathétiquement accessible ou semble le devenir, et il se rapproche alors de nous, tantôt l'intrigue dissimulée par la nuit ou disparue dans les ellipses et dans les lacunes du texte nous sépare de lui, de son souvenir d'enfance, de ses secrètes interrogations et des tumultueux mouvements de son âme dont nous ne pouvons contempler, sur le fond élargi de la lune et de la forge, que le reflet insistant, obsédant mais incertain.

Cette incertitude subjective nous poursuivra toute notre vie. Une brume bleue tombe sur le "Romance » et sur son sujet. Le chemin tracé par les trente-six octosyllabes est une allée qui me conduit vers la maison de mon désir, je m'approche lentement de ce savant composé d'amour, de sexe, de deuil et de mort, puis à l'instant même où l'action décisive d'entrer dans la maison va se produire, la saisie esthétique de ce sujet poétique, que quelque chose toujours m'empêchait de voir, devient définitivement impossible :

je ne suis pas assez belle pour lui.

Au moins avons-nous compris que le sujet d'écriture n'est pas dans le tréfonds sentimental et psychologique d'Eugénie, pas plus que dans les tourments oedipiens de l'enfant à la forge. Il serait plutôt cette sarabande d'images, de sons et de représentations sur fond de pan de mur ensoleillé ou de forge et de paysage andalou éclairés par la lune, impossible à saisir autrement que dans la littéralité mouvante et savante du roman et du "Romance ". Sagesse d'une définition du sujet d'écriture qui nous renvoie à la splendeur de l'objet esthétique et de l'événement qui le traverse, esthétisation du sujet qui nous le rend sinon connaissable du moins doux à savourer, apothéose du sujet dans la littéralité de ses textes successifs qui sont sa seule réalité, son seul lieu d'existence et de plénitude.

Jamais je ne serai assez belle pour lui. 